\section{BRAZIULIAN JOURNAL \\ OF MEDICAL AND BIOLOGICAL RESF.ARCH}

www.bjournal.com.br
ISSN 0100-879X

Volume 43 (04) 325-408

April 2010

BIOMEDICAL SCIENCES

AND

CLINICAL INVESTIGATION

Braz J Med Biol Res, March 2010, Volume 43(4) 390-396

Kinetics of cardiac and vascular remodeling by spontaneously hypertensive rats after discontinuation of long-term captopril treatment

W.A. Rocha, W. Lunz, M.P. Baldo, E.B. Pimentel, E.M. Dantas, S.L. Rodrigues and J.G. Mill

The Brazilian Journal of Medical and Biological Research is partially financed by
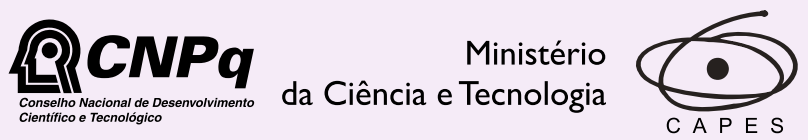

Ministério da Educação

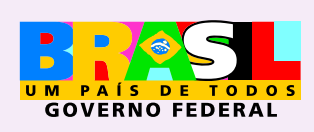

Institutional Sponsors 


\title{
Kinetics of cardiac and vascular remodeling by spontaneously hypertensive rats after discontinuation of long-term captopril treatment
}

\author{
W.A. Rocha1 ${ }^{1}$, W. Lunz ${ }^{1,2}$, M.P. Baldo ${ }^{1}$, E.B. Pimentel ${ }^{1}$, E.M. Dantas ${ }^{1}$, \\ S.L. Rodrigues ${ }^{1}$ and J.G. Mill ${ }^{1}$ \\ ${ }^{1}$ Departamento de Ciências Fisiológicas, ${ }^{2}$ Centro de Educação Física e Desportos, \\ Universidade Federal do Espírito Santo, Vitória, ES, Brasil
}

\begin{abstract}
Angiotensin-converting enzyme inhibitors reduce blood pressure and attenuate cardiac and vascular remodeling in hypertension. However, the kinetics of remodeling after discontinuation of the long-term use of these drugs are unknown. Our objective was to investigate the temporal changes occurring in blood pressure and vascular structure of spontaneously hypertensive rats (SHR). Captopril treatment was started in the pre-hypertensive state. Rats (4 weeks) were assigned to three groups: SHR-Cap $(\mathrm{N}=51)$ treated with captopril $(1 \mathrm{~g} / \mathrm{L})$ in drinking water from the 4th to the 14th week; SHR-C $(\mathrm{N}=48)$ untreated SHR; Wistar $(N=47)$ control rats. Subgroups of animals were studied at 2, 4, and 8 weeks after discontinuation of captopril. Direct blood pressure was recorded in freely moving animals after femoral artery catheterism. The animals were then killed to determine left ventricular hypertrophy (LVH) and the aorta fixed at the same pressure measured in vivo. Captopril prevented hypertension (105 \pm 3 vs $136 \pm 5 \mathrm{mmHg}), \mathrm{LVH}(2.17 \pm 0.05 \mathrm{vs} 2.97 \pm 0.14 \mathrm{mg} / \mathrm{g}$ body weight) and the increase in cross-sectional area to luminal area ratio of the aorta $\left(0.21 \pm 0.01\right.$ vs $\left.0.26 \pm 0.02 \mu \mathrm{m}^{2}\right)$ (SHR-Cap vs SHR-C). However, these parameters increased progressively after discontinuation of captopril (22nd week: $141 \pm 2 \mathrm{mmHg}, 2.50 \pm 0.06 \mathrm{mg} / \mathrm{g}, 0.27 \pm 0.02 \mu \mathrm{m}^{2}$ ). Prevention of the development of hypertension in SHR by using captopril during the prehypertensive period prevents the development of cardiac and vascular remodeling. Recovery of these processes follows the kinetic of hypertension development after discontinuation of captopril.
\end{abstract}

Key words: SHR; Cardiac hypertrophy; Aorta remodeling; ACE inhibition; Captopril

\section{Introduction}

Hypertension is both a complex disease and an important risk factor for other cardiovascular outcomes, such as sudden death, stroke, myocardial infarction, heart failure, and renal disease (1-3). The spontaneously hypertensive rat (SHR) is considered to be the best animal model for studies of essential hypertension in humans. The kinetics of blood pressure changes in these animals can be divided into three phases. In the pre-hypertensive phase (up to the 4th postnatal week), their blood pressure is similar to that of control normotensive rats. In the labile-hypertension phase (5th to 14th week of age) there is a progressive increase of blood pressure. Finally, in the established-hypertension phase (starting from the 15th week) steady and high blood pressure levels are observed until the end of life (4). This classification, however, has not been consensual $(5,6)$.
Captopril was the first angiotensin-converting enzyme (ACE) inhibitor clinically used in hypertensive patients and has been widely used in experimental and clinical studies, showing that its long-term use normalizes blood pressure, prevents the development of cardiac hypertrophy and vascular remodeling, and decreases mortality and morbidity $(2,6-11)$. Some studies have shown that the long-term use of captopril in SHR in the prehypertensive phase is able not only to prevent the development of high blood pressure levels and cardiac hypertrophy, but also to maintain low blood pressure levels even after discontinuation of its use $(2,12)$. These findings differ from those obtained with other antihypertensive drugs administered to SHR such as $\beta$-blockers, vasodilators and calcium antagonists, whose discontinuation results in a return of blood pressure levels

Correspondence: J.G. Mill, Departamento de Ciências Fisiológicas, Universidade Federal do Espírito Santo, Av. Marechal Campos, 1468, 29042-751 Vitória, ES, Brasil. Fax: +55-27-3335-7330. E-mail: josegmill@gmail.com

Received September 6, 2009. Accepted March 11, 2010. Available online April 5, 2010. Published April 12, 2010. 
equivalent to those seen in untreated animals $(13,14)$. Freslon and Giudicelli (12), for example, observed that treatment with captopril and hydralazine normalized blood pressure. However, only the animals treated with captopril (100 $\mathrm{mg} \cdot \mathrm{kg}^{-1} \cdot \mathrm{day}^{-1}$ ) maintained normal blood pressure levels for 7 weeks after treatment interruption. Other studies have shown that when captopril was given to SHR in the prehypertensive phase $(8,15)$ or since intrauterine life $(16,17)$, the arterial pressure and cardiac mass were maintained within normal limits for a long period of time after birth even after discontinuation of captopril. It has been argued that the use of captopril during the prehypertensive phase may prevent the hypertrophy of arterial smooth muscle, thus preventing the development of hypertension in later phases of life. Thus, our objective in this study was to compare the patterns of the remodeling process occurring in the heart and in the aorta of SHR treated with captopril beginning in the prehypertensive phase.

\section{Material and Methods}

\section{Animals and experimental groups}

Rats were divided into three groups at the age of 4 weeks: a) SHR-Cap ( $\mathrm{N}=51)$, SHR treated with captopril; b) SHR-C ( $\mathrm{N}=48)$, untreated control SHR, and c) untreated Wistar rats $(\mathrm{N}=47)$. Sex was balanced within each group. The animals had free access to water and rat chow. Captopril was dissolved in the drinking water $(1 \mathrm{~g} / \mathrm{L})$ and offered to the SHR-Cap group from the 4th to the 14th week after birth. Subgroups ( $N=11$ to 15 ) of SHR-Cap, SHR-C and Wistar animals were studied at the ages of $14,16,18$, and 22 weeks to measure blood pressure and to determine cardiac and vascular remodeling parameters. All animals were maintained in rooms with controlled temperature (20$25^{\circ} \mathrm{C}$ ) and a 12:12-h dark/light cycle. All procedures were according to the Guide for the Care and Use of Laboratory Animals (NIH Publication No. 85-23, revised 1996), and the project was approved by our Committee of Ethics on Animal Research (Protocol \#021/2007) in the Universidade Federal do Espírito Santo.

\section{Blood pressure record}

A polyethylene catheter (PE10 coupled to PE50) was inserted into the left femoral artery under chloral hydrate (400 $\mathrm{mg} / \mathrm{kg}$ ) anesthesia and fixed to the dorsal neck region. Blood pressure was recorded in freely moving and unrestrained animals $12-16 \mathrm{~h}$ later by connecting the arterial catheter to a pressure transducer (Statham, model P23AA, USA) coupled to an amplifier (FUNBEC, model MP100, Brazil) and to a chart recorder (FUNBEC, model RG300). Mean arterial pressure (MAP) and heart rate were determined electronically.

\section{Arterial measurements}

After blood pressure recording the animals were anes- thetized again and the heart was rapidly removed and rinsed in physiological saline. The ventricles were separated and weighed. Hypertrophy of the cardiac chambers was determined by the chamber weight to body weight ratio. A metal needle was attached to the aortic arch and blood from the arterial tree was washed out with warmed $\left(37^{\circ} \mathrm{C}\right)$ saline. The needle was then connected to a perfusion system containing Bouin solution. The perfusion pressure was adjusted to the same value of the MAP measured in vivo in order to maintain as much as possible the vascular shape without deformation. After 30-min perfusion, a 1-cm fragment of the aorta was removed at the level of the diaphragm and stored for $24 \mathrm{~h}$ in Bouin solution. The aortic ring was embedded in paraffin. Slices (5 $\mu \mathrm{m}$, Rotary Microtome, American Optical, Model 820, USA) were stained with hematoxylineosin and used for histological measurements obtained with dedicated software (Leica EWS 2100, Germany) at 40 to $120 X$ magnification (Olympus AX-70 microscope, Japan). Four transverse slices were examined from each aorta and the mean of these measurements was used as the representative value for each animal. A minimum of 4 animals were studied in each group and age. The artery was considered to have an elliptical shape and the areas from the lumen and media were calculated as previously described (18). The circumferential wall tension (dyn/cm) was calculated as MAP $x$ artery radius, and the wall stress $\left(\mathrm{dyn} / \mathrm{\mu m}^{2}\right)$ as [(MAP x luminal area $\left.(\mathrm{LA})\right) /$ transverse section area (TSA)].

\section{Statistical analysis}

Data are reported as means \pm SEM. One-way analysis of variance (ANOVA) followed by the Tukey post hoc test was used to compare means. Statistical analysis was performed using the GraphPad Prism software (USA) and statistical significance was set at $P<0.05$.

\section{Results}

The body weight of the Wistar group was greater than that of both SHR groups. A tendency to lower body weight was observed in the SHR-Cap group, and the difference between the untreated and treated SHR groups became progressively smaller after captopril was removed from drinking water (data not shown).

Blood pressure recorded in awake and unrestrained animals is shown in Figure 1. As expected, captopril fully prevented the development of hypertension in the SHRCap group, since after 10 weeks of treatment (4th to 14th week) the MAP of the SHR-Cap group was similar to that of the Wistar group (105 \pm 3 vs $107 \pm 3 \mathrm{mmHg}$; P > 0.05). After discontinuation of captopril (14th week), a progressive increase in blood pressure was observed in SHR-Cap animals (Figure 1).

The variation of the left ventricle weight to body weight ratio (LV/BW) in the Wistar, SHR, and SHR-Cap groups 
at the 14th, 16th, 18th, and 22nd week of postnatal life is presented in Figure 2. In the groups studied just at the end of captopril treatment (14th week) the LV/BW ratio of SHR-Cap $(2.17 \pm 0.05 \mathrm{mg} / \mathrm{g})$ was similar to that of Wistar rats $(2.10 \pm 0.04 \mathrm{mg} / \mathrm{g})$, but was significantly lower in both groups compared to the SHR-C group $(2.97 \pm 0.14 \mathrm{mg} / \mathrm{g}$; $P$ $<0.05$ ), indicating that captopril prevented the left ventricle hypertrophy observed in untreated SHR animals. After captopril interruption, there was a progressive increase of the LV/BW ratio in the SHR-Cap group (16th: $2.36 \pm 0.09$, 18th: $2.44 \pm 0.06,22 \mathrm{nd}: 2.50 \pm 0.06 \mathrm{mg} / \mathrm{g})$ compared to the Wistar group (16th: $2.03 \pm 0.04,18$ th: $1.93 \pm 0.06,22 \mathrm{nd}$ : $1.89 \pm 0.16 \mathrm{mg} / \mathrm{g})$. These data show that development of left ventricular hypertrophy in untreated SHR depends on the presence of high blood pressure or at least in part on ACE activation as demonstrated by others $(19,20)$. The right ventricle weight to body weight ratio did not differ between the SHR-Cap (14th week: $0.67 \pm 0.04,16$ th week: $0.68 \pm$
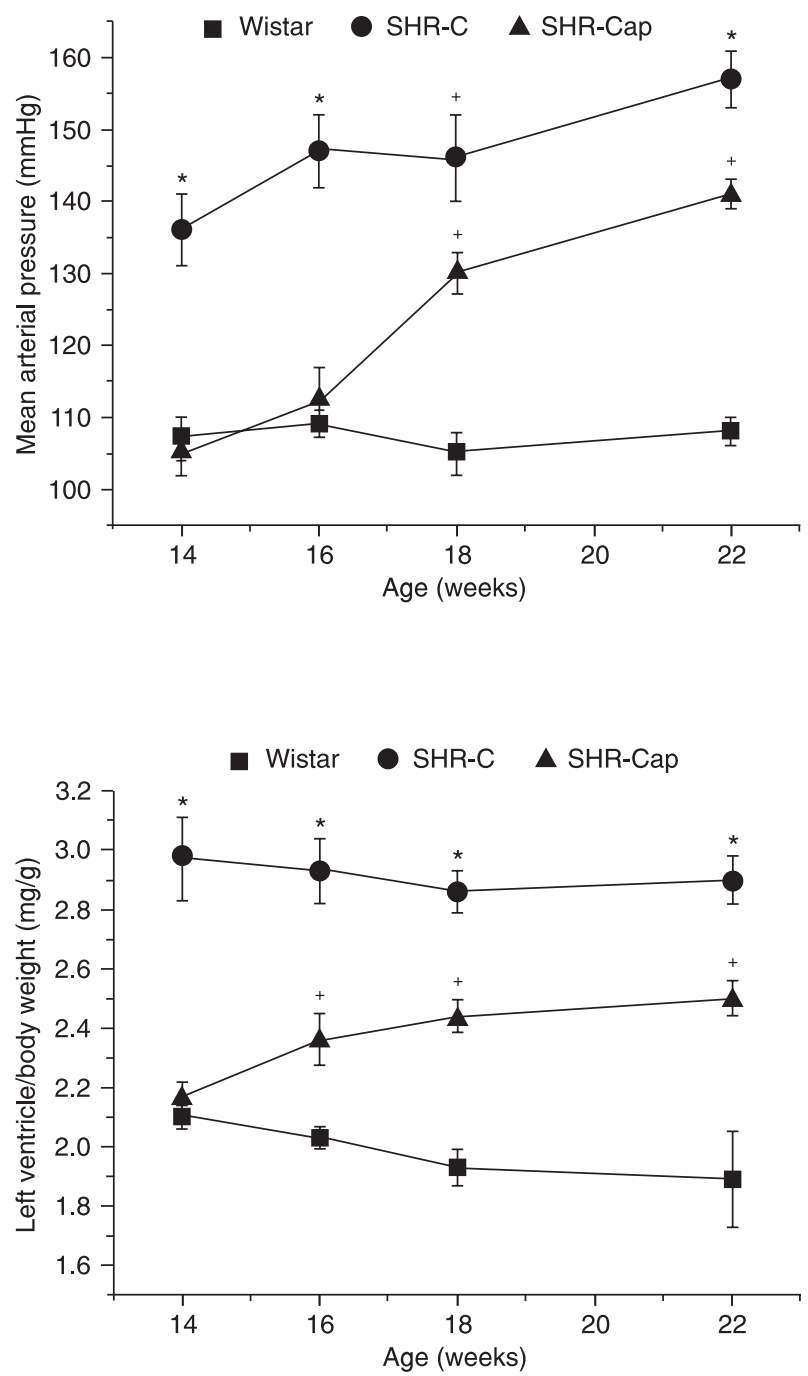

$0.03,18$ th week: $0.70 \pm 0.08,22$ nd week: $0.65 \pm 0.03 \mathrm{mg} / \mathrm{g}$ ) and SHR-C groups (14th week: $0.69 \pm 0.02$, 16th week: $0.70 \pm 0.03$, 18th week: $0.69 \pm 0.03,22$ nd week: $0.65 \pm$ $0.02 \mathrm{mg} / \mathrm{g}$ ) at any age.

To determine whether chronic captopril use was able to promote morphological changes in the aorta, the media TSA and LA were measured in the animal groups at different ages. The TSA/LA ratio was calculated and also compared. Similar to the blood pressure findings, the increase of the TSA/LA ratio in the aorta was fully prevented by captopril treatment. At the 14th week the value for the SHR-Cap group was similar $(0.21 \pm 0.01)$ to that of the Wistar group $(0.19 \pm$ $0.01)$, but significantly lower than that of the untreated SHR group ( $0.26 \pm 0.02$; Figure 3$)$, indicating the development of typical artery remodeling secondary to hypertension. After discontinuation of captopril treatment, a gradual increase of the TSA/LA ratio was observed in the SHR-Cap group (16th week: $0.23 \pm 0.01,18$ th week: $0.25 \pm 0.01,22$ nd week:

Figure 1. Mean arterial pressure (MAP) of the Wistar, SHR-C and SHR-Cap groups in the 14th, 16th, 18th, and 22nd weeks of post-uterine life. SHR-Cap = group of spontaneously hypertensive rats (SHR) treated with $1 \mathrm{~g} / \mathrm{L}$ captopril in drinking water $(\mathrm{N}=$ 12-15); SHR-C = group of untreated SHR $(\mathrm{N}=11-13)$; Wistar $=$ group of untreated Wistar rats $(\mathrm{N}=11-12)$. Data are reported as means \pm SEM. ${ }^{*} \mathrm{P}<0.05$ vs Wistar and SHR-Cap; ${ }^{+} \mathrm{P}<0.05$ vs Wistar (one-way ANOVA).

Figure 2. Left ventricle mass to body weight ratio (LV/BW) of the Wistar, SHR-C and SHR-Cap groups in the 14th, 16th, 18th, and 22nd weeks of post-uterine life. SHR-Cap = group of spontaneously hypertensive rats (SHR) treated with $1 \mathrm{~g} / \mathrm{L}$ captopril in drinking water $(\mathrm{N}=12-15)$; SHR-C = group of untreated SHR $(\mathrm{N}=$ 11-13); Wistar = group of untreated Wistar rats $(\mathrm{N}=11-12)$. Data are reported as means \pm SEM. ${ }^{*} \mathrm{P}<0.05$ vs Wistar and SHR-Cap; ${ }^{+} \mathrm{P}<0.05$ vs Wistar (one-way ANOVA). 
$0.27 \pm 0.01)$ while a stable TSA/LA ratio was observed in the Wistar group (16th week: $0.18 \pm 0.01$, 18th week: 0.19 $\pm 0.01,22$ nd week: $0.19 \pm 0.01$; Figure 3 ).

After discontinuation of captopril, TSA increased by 11,12 and $19 \%$ at the 16 th, 18 th, and 22 nd week, respectively. However, the aorta LA underwent a reduction of 5 and $1 \%$ in the 18 th and 22 nd week groups, respectively. These results suggest that captopril treatment has a more important participation in the remodeling of the medial cross-sectional area. Additionally, as can be observed in Figures 1 and 2, MAP and LV/BW increased progressively after the discontinuation of captopril treatment. The increase in MAP at the 16th, 18th, and 22nd week was 7,24 , and $34 \%$, and the increase of the LV/BW ratio was 9,12 , and
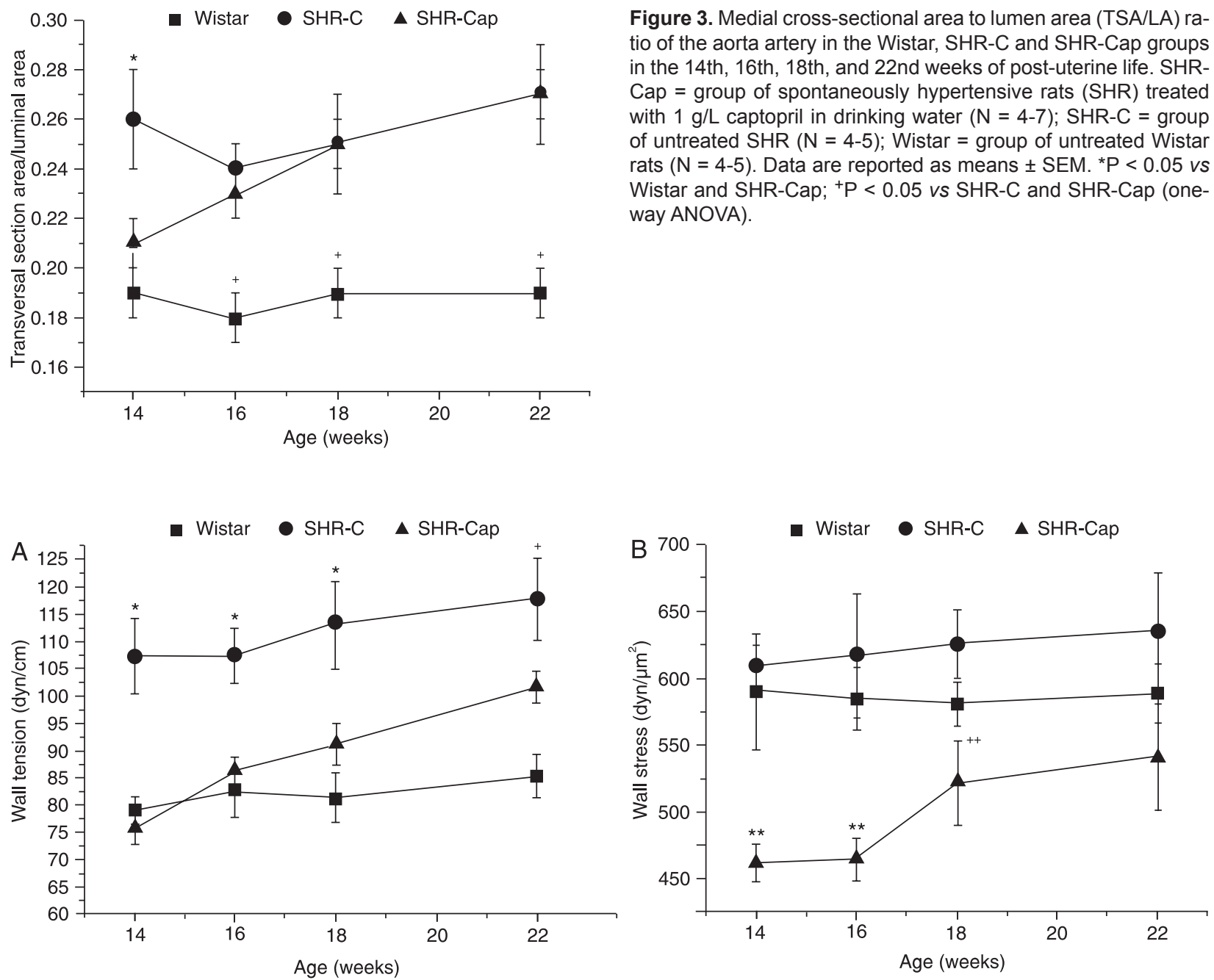

Figure 4. Aorta wall tension $(A)$ and stress $(B)$ in the Wistar $(N=4-5)$, SHR-C $(N=4-5)$ and SHR-Cap $(N=4-7)$ groups in the 14th, 16th, 18th, and 22nd weeks of post-uterine life. Data are reported as means \pm SEM. SHR-C = group of untreated spontaneously hypertensive rats (SHR); SHR-Cap = group of SHR treated with $1 \mathrm{~g} / \mathrm{L}$ captopril in drinking water. ${ }^{*} \mathrm{P}<0.05$ vs Wistar and SHR-Cap; ${ }^{+} \mathrm{P}$ $<0.05$ vs Wistar; ${ }^{*} \mathrm{P}<0.05$ vs Wistar and SHR-C; ${ }^{++} \mathrm{P}<0.05$ vs SHR-C (one-way ANOVA).

Figure 3. Medial cross-sectional area to lumen area (TSA/LA) ratio of the aorta artery in the Wistar, SHR-C and SHR-Cap groups in the 14th, 16th, 18th, and 22nd weeks of post-uterine life. SHRCap = group of spontaneously hypertensive rats (SHR) treated with $1 \mathrm{~g} / \mathrm{L}$ captopril in drinking water ( $\mathrm{N}=4-7)$; SHR-C = group of untreated SHR $(\mathrm{N}=4-5)$; Wistar = group of untreated Wistar rats $(\mathrm{N}=4-5)$. Data are reported as means \pm SEM. ${ }^{*} \mathrm{P}<0.05$ vs Wistar and SHR-Cap; ${ }^{+} \mathrm{P}<0.05$ vs SHR-C and SHR-Cap (oneway ANOVA).

$5 \%$, respectively.

4. After the discontinuation of captopril treatment, the wall tension of the SHR-Cap group showed a progressive 2.64, 18th: $91.09 \pm 3.76$, and 22 nd week: $101.36 \pm 2.87$ $\mathrm{dyn} / \mathrm{cm})$, although it was not statistically different from the values measured in the Wistar group (14th week: $78.90 \pm$ and 22nd week: $85.22 \pm 4.01 \mathrm{dyn} / \mathrm{cm})$. The SHR-C group, however, presented significantly higher $(P<0.05)$ values (14th week: $107.17 \pm 7.03,16$ th week: $107.35 \pm 5.18,18$ th
week: $112.99 \pm 8.07$, and 22 nd week: $117.73 \pm 7.6 \mathrm{dyn} / \mathrm{cm}$ ) than those measured in the SHR-Cap and Wistar groups, 
except at the $22 \mathrm{nd}$ week when no significant difference was observed between the SHR-Cap and SHR-C groups. In the 14th week, the SHR-Cap group presented a lower value of wall stress $\left(462.00 \pm 14.01 \mathrm{dyn} / \mathrm{\mu m}^{2} ; \mathrm{P}<0.05\right)$ than the SHR-C $\left(608.59 \pm 16.54 \mathrm{dyn} / \mu^{2}\right)$ and Wistar groups (589.90 $\left.\pm 43.34 \mathrm{dyn} / \mu \mathrm{m}^{2}\right)$. After the discontinuation of captopril the wall stress of the SHR-Cap group increased progressively, so that in the 22nd week there were no differences (541.15 $\left.\pm 39.41 \mathrm{dyn} / \mu^{2}{ }^{2}\right)$ compared to the SHR-C (635.17 \pm 44.04 $\left.\mathrm{dyn} / \mu \mathrm{m}^{2}\right)$ and Wistar (588.26 $\left.\pm 22.43 \mathrm{dyn} / \mu \mathrm{m}^{2}\right)$ groups.

\section{Discussion}

The observations reported here provide support to the hypothesis that vascular remodeling in SHR is not genetically mediated, but depends strictly on blood pressure increase during the life of these animals. Some studies have shown that long-term captopril treatment not only reduces high blood pressure levels in hypertension $(2,6,8,10)$ but also reverses both the cardiac and vascular smooth muscle hypertrophy (6). In the present study, we demonstrated that captopril treatment starting from the prehypertensive phase fully prevents the development of hypertension as well as cardiac hypertrophy and aorta remodeling. It has been argued that the precocious use of ACE inhibitors in hypertension might interfere with some anatomical and/or functional parameters necessary to prevent age-dependent blood pressure increase $(21,22)$.

In the present study, we made serial observations related not only to blood pressure, but also to cardiac hypertrophy and aortic remodeling for 8 weeks after discontinuation of captopril. With the use of direct assessment of blood pressure and histological measurements, our findings did not confirm previous observations that precocious use of ACE inhibitors can prevent hypertension development later.

Our observations related to the reduction of blood pressure and prevention of cardiac hypertrophy and vascular remodeling in SHR under captopril treatment are similar to those reported by others $(8,15-18)$. However, differently from other studies $(8,16,17)$, after interruption of captopril treatment we observed a progressive increase of blood pressure and a parallel increase of left ventricular mass and aortic remodeling. Thus, Harrap et al. (8) treated young SHR with perindopril and observed that blood pressure was reduced significantly during treatment, rose when treatment was stopped, but plateaued significantly below the blood pressure levels of untreated SHR. Fifteen weeks after perindopril discontinuation, treated SHR animals also presented less left ventricular weight compared to untreated SHR.

In our study, captopril was initiated 4 weeks after birth. Wu and Berecek (17) did not analyze cardiac remodeling, but they showed that when the dams were treated with ACE inhibitors their offspring presented lower blood pressure levels. According to these investigators, the early use of captopril in SHR may change the expression or function of angiotensin II receptors, determining long-term effects on blood pressure regulation. However, short-term treatment of young SHR with perindopril (8) was sufficient to reduce systolic blood pressure and to maintain it for several weeks after discontinuation of treatment. We did not observe this finding in our study, suggesting that drugspecific effects of different ACE inhibitors may account for these differences.

Our data show clearly that blood pressure progressively increased as captopril was withdrawn from pretreated SHR animals. Left ventricular hypertrophy and aortic remodeling also develop with time. However, cardiac hypertrophy appears before blood pressure increase, suggesting that myocyte hypertrophy may be independent, at least in part, on the increase in blood pressure. In fact, ACE inhibition can attenuate cardiac hypertrophy independent of a change in blood pressure (23), suggesting that this effect may be secondary to the blockade of the local renin-angiotensinaldosterone system in the heart. Moreover, it is well known that SHR have increased sympathetic activity (24). Veliotes et al. (25) reported that cardiac hypertrophy and dysfunction induced by chronic $\beta$-adrenergic stimulation are mediated by the activation of the local renin-angiotensin-aldosterone system in the heart, a fact previously observed in rats treated with isoproterenol (26). Thus, the activation of the local renin-angiotensin-aldosterone system in the heart may accelerate the development of cardiac hypertrophy before the rise in blood pressure.

We also observed a reduction of the TSA/LA ratio with long-term captopril treatment, but after discontinuation of treatment there was a progressive increase of the aorta TSA/ LA ratio. It is worth noting that in our study the aorta fixation process was performed in such a way as to maintain the internal forces at a value similar to that occurring in vivo, since the vessel was fixed at the same pressure as mean blood pressure. This protocol allows the maintenance of arterial shape and a more reliable evaluation of vascular remodeling. Additionally, we observed that the prevention of aortic remodeling in SHR treated with captopril mainly affected the media layer, and not the LA. Giummelly et al. (6) also observed that 6 months of SHR treatment with captopril plus hydrochlorothiazide reduced the medial crosssectional area but not the internal diameter of the thoracic aorta. The internal diameter was altered only after 1 year of treatment. Perindopril treatment of SHR from 6 to 10 weeks of age reduced arterial pressure, relative cardiac mass, media thickness and the media/lumen ratio of mesenteric vessels by the $32 \mathrm{nd}$ week, but no differences was found in the lumen diameter of these vessels (8). These results contradict the suggestion that arterial lumen reduction is the main factor related to the increase in arterial TSA/LA ratio in hypertension (27).

Aorta wall tension was normalized by captopril treatment and increased progressively after treatment discontinuation. Since wall tension is the product of arterial pressure 
to arterial radius it is expected that this index may follow the behavior of arterial pressure. As expected, the measure of the aorta wall stress obtained by the ratio between wall tension and TSA presented lower values in the SHR-Cap group in the 14th week, with a progressive increase in the subsequent weeks, following a time course similar to that observed for mean arterial pressure.

It is noteworthy that, although our data differ from those obtained in some experimental studies, because SHR used in our experiment recovered high blood pressure levels as captopril use was discontinued, clinical findings support our hypothesis that discontinuation of ACE inhibitors leads to a return of hypertension (28-30). Thus, our data clearly demonstrate that vascular remodeling in hypertension is a dynamic process and that antihypertensive drugs, i.e., ACE inhibitors, only attenuate the progression of remodeling, with the consequent need to maintain therapy.

The fact that male and female rats were pooled in each group is a limitation of our study. In fact, a comparison between sexes showed no difference. However, the number

\section{References}

1. Hillege HL, Girbes AR, de Kam PJ, Boomsma F, de Zeeuw $D$, Charlesworth $A$, et al. Renal function, neurohormonal activation, and survival in patients with chronic heart failure. Circulation 2000; 102: 203-210.

2. Kost CK Jr, Li P, Jackson EK. Blood pressure after captopril withdrawal from spontaneously hypertensive rats. Hypertension 1995; 25: 82-87.

3. Smith PG, Poston CW, Mills E. Ontogeny of neural and nonneural contributions to arterial blood pressure in spontaneously hypertensive rats. Hypertension 1984; 6: 54-60.

4. Okamoto K, Aoki K. Development of a strain of spontaneously hypertensive rats. Jpn Circ J 1963; 27: 282-293.

5. Dickhout JG, Lee RM. Blood pressure and heart rate development in young spontaneously hypertensive rats. $\mathrm{Am} \mathrm{J}$ Physiol 1998; 274: H794-H800.

6. Giummelly P, Lartaud-Idjouadiene I, Marque V, Niederhoffer $\mathrm{N}$, Chillon JM, Capdeville-Atkinson C, et al. Effects of aging and antihypertensive treatment on aortic internal diameter in spontaneously hypertensive rats. Hypertension 1999; 34: 207-211.

7. Bicket DP. Using ACE inhibitors appropriately. Am Fam Physician 2002; 66: 461-468.

8. Harrap SB, Van der Merwe WM, Griffin SA, Macpherson $F$, Lever AF. Brief angiotensin converting enzyme inhibitor treatment in young spontaneously hypertensive rats reduces blood pressure long-term. Hypertension 1990; 16: 603614.

9. Mathew J, Sleight P, Lonn E, Johnstone D, Pogue J, Yi Q, et al. Reduction of cardiovascular risk by regression of electrocardiographic markers of left ventricular hypertrophy by the angiotensin-converting enzyme inhibitor ramipril. Circulation 2001; 104: 1615-1621.

10. Paull JR, Widdop RE. Persistent cardiovascular effects of chronic renin-angiotensin system inhibition following withdrawal in adult spontaneously hypertensive rats. J Hyper- of animals used in the study was too small to detect possible differences.

The present study shows that captopril treatment of SHR for 10 weeks starting from the prehypertensive phase fully prevented blood pressure increase and aorta remodeling. These effects, however, disappeared when treatment was stopped and 8 weeks later the beneficial effects of blood pressure control (reduction of cardiac hypertrophy, reduction of arterial stress) were reduced. Our findings in SHR agree with clinical observations of hypertensive patients, in which the beneficial effects of long-term use of ACE inhibitors also disappear after weeks or months after treatment interruption.

\section{Acknowledgments}

Research supported by CNPq (\#501599/2008-8 and \#302296/2008-5) and Fundação de Amparo à Pesquisa do Espírito Santo (FAPES/Pronex \#35884886).

tens 2001; 19: 1393-1402.

11. Sharma JN, Kesavarao U. Effect of captopril on urinary kallikrein, blood pressure and myocardial hypertrophy in diabetic spontaneously hypertensive rats. Pharmacology 2002; 64: 196-200.

12. Freslon JL, Giudicelli JF. Compared myocardial and vascular effects of captopril and dihydralazine during hypertension development in spontaneously hypertensive rats. Br J Pharmacol 1983; 80: 533-543.

13. Sevilla MA, Voces F, Carron R, Guerrero El, Ardanaz N, Román LS, et al. Amlodipine decreases fibroses and cardiac hypertrophy in spontaneously hypertensive rats: persistent effects after withdrawal. Life Sci 2004; 75: 881-891.

14. Guerrero El, Ardanaz N, Sevilla MA, Arévalo MA, Montero MJ. Cardiovascular effects of nebivolol in spontaneously hypertensive rats persist after treatment withdrawal. $\mathrm{J} \mathrm{Hy}$ pertens 2006; 24: 151-158.

15. Cadilhac M, Giudicelli JF. Myocardial and vascular effects of perindopril, a new converting enzyme inhibitor, during hypertension development in spontaneously hypertensive rats. Arch Int Pharmacodyn Ther 1986; 284: 114-126.

16. Chen S, Su J, Wu K, Hu W, Gardner DG, Chen D. Early captopril treatment prevents hypertrophy dependent gene expression in hearts of SHR. Am J Physiol 1998; 274: R1511-R1517.

17. Wu JN, Berecek KH. Prevention of genetic hypertension by early treatment of spontaneously hypertensive rats with the angiotensin converting enzyme inhibitor captopril. Hypertension 1993; 22: 139-146.

18. Schiffrin EL, Hayoz D. How to assess vascular remodelling in small and medium-sized muscular arteries in humans. $J$ Hypertens 1997; 15: 571-584.

19. Wright JW, Mizutani S, Harding JW. Pathways involved in the transition from hypertension to hypertrophy to heart failure. Heart Fail Rev 2008; 13: 367-375. 
20. Li L, Yi-Ming W, Li ZZ, Zhao L, Yu YS, Li DJ. Local RAS and inflammatory factors are involved in cardiovascular hypertrophy in spontaneously hypertensive rats. Pharmacol Res 2008; 58: 196-201.

21. Celik T, Iyisoy A, Acikel C, Yuksel C, Celik M, Yaman H, et al. The comparative effects of metoprolol and perindopril on aortic elasticity in young patients with prehypertension. Blood Press Monit 2008; 13: 169-176.

22. Egan BM, Nesbitt SD, Julius S. Prehypertension: should we be treating with pharmacologic therapy? Ther Adv Cardiovasc Dis 2008; 2: 305-314.

23. Avanza AC Jr, El Aouar LM, Mill JG. Reduction in left ventricular hypertrophy in hypertensive patients treated with enalapril, losartan or the combination of enalapril and losartan. Arq Bras Cardiol 2000; 74: 103-117.

24. Yajima $Y$, Ito S, Komatsu K, Tsukamoto K, Matsumoto K, Hirayama A. Enhanced response from the caudal pressor area in spontaneously hypertensive rats. Brain Res 2008; 1227: 89-95.

25. Veliotes DG, Woodiwiss AJ, Deftereos DA, Gray D, Osadchii $\mathrm{O}$, Norton GR. Aldosterone receptor blockade prevents the transition to cardiac pump dysfunction induced by beta-adrenoreceptor activation. Hypertension 2005; 45: 914-920.
26. Busatto VC, Cunha V, Cicilini MA, Mill JG. Differential effects of isoproterenol on the activity of angiotensin-converting enzyme in the rat heart and aorta. Braz J Med Biol Res 1999; 32: $355-360$

27. Intengan HD, Schiffrin EL. Structure and mechanical properties of resistance arteries in hypertension: role of adhesion molecules and extracellular matrix determinants. Hypertension 2000; 36: 312-318.

28. Nelson MR, Reid CM, Krum H, Muir T, Ryan P, McNeil JJ. Predictors of normotension on withdrawal of antihypertensive drugs in elderly patients: prospective study in second Australian national blood pressure study cohort. BMJ 2002; 325: 815.

29. Nelson M, Reid C, Krum H, McNeil J. A systematic review of predictors of maintenance of normotension after withdrawal of antihypertensive drugs. Am J Hypertens 2001; 14: 98105.

30. Takata Y, Yoshizumi T, Ito Y, Ueno M, Tsukashima A, Iwase $M$, et al. Comparison of withdrawing antihypertensive therapy between diuretics and angiotensin converting enzyme inhibitors in essential hypertensives. Am Heart J 1992; 124: 1574-1580. 